\title{
Pseudomonas aeruginosa prioritizes detoxification of hydrogen peroxide over nitric oxide
}

\author{
Darshan M. Sivaloganathan ${ }^{1}$ and Mark P. Brynildsen ${ }^{2^{*}}$ (1)
}

\begin{abstract}
Objective: Bacteria are exposed to multiple concurrent antimicrobial stressors within phagosomes. Among the antimicrobials produced, hydrogen peroxide and nitric oxide are two of the most deleterious products. In a previous study, we discovered that when faced with both stressors simultaneously, Escherichia coli prioritized detoxification of hydrogen peroxide over nitric oxide. In this study, we investigated whether such a process was conserved in another bacterium, Pseudomonas aeruginosa.

Results: P. aeruginosa prioritized hydrogen peroxide detoxification in a dose-dependent manner. Specifically, hydrogen peroxide detoxification was unperturbed by the presence of nitric oxide, whereas larger doses of hydrogen peroxide produced longer delays in nitric oxide detoxification. Computational modelling revealed that the rate of nitric oxide consumption in co-treated cultures was biphasic, with cells entering the second phase of detoxification only after hydrogen peroxide was eliminated from the culture.
\end{abstract}

Keywords: Fhp, Catalase, Hydroperoxide reductase, $\mathrm{NO}, \mathrm{H}_{2} \mathrm{O}_{2}$, Antimicrobial, Phagosome

\section{Introduction}

Phagosomes are complex environments in which bacteria are exposed concurrently to a multitude of stressors $[1-3]$. Among these antimicrobials are nitric oxide (NO) and hydrogen peroxide $\left(\mathrm{H}_{2} \mathrm{O}_{2}\right)[1,3,4]$. Both $\mathrm{NO}$ and $\mathrm{H}_{2} \mathrm{O}_{2}$ rapidly diffuse across bacterial membranes and are capable of damaging a diverse array of biomolecules within cells [5-9]. NO can directly damage iron-sulfur clusters in proteins and block cellular respiration by reversibly binding heme groups [6, 7]. Moreover, NO can react with oxygen and superoxide to produce even more toxic molecules, termed reactive nitrogen species (RNS), that can cause lipid peroxidation, DNA deamination, and

\footnotetext{
*Correspondence: mbrynild@princeton.edu

${ }^{2}$ Department of Chemical and Biological Engineering, Princeton University, Princeton, NJ, USA

Full list of author information is available at the end of the article
}

nitrosylation of thiols and tyrosines [6, 9]. Similarly, $\mathrm{H}_{2} \mathrm{O}_{2}$ can damage proteins by disrupting iron-sulfur clusters and reacting with specific amino acid residues, such as cysteine and methionine $[5,8]$. Further, $\mathrm{H}_{2} \mathrm{O}_{2}$ can react with ferrous iron to generate hydroxyl radical, which is an even more deleterious species that is capable of reacting with a wide array of biomolecules within cells, including nucleic acids, lipids, sugars, and amino acids $[8,10]$.

Bacteria have evolved detoxification systems to combat these stressors. For example, Escherichia coli possess an NO dioxygenase ( $\mathrm{Hmp}$ ) and an NO reductase (NorV) to eliminate $\mathrm{NO}$ under aerobic and anaerobic conditions, respectively $[6,9]$. To detoxify $\mathrm{H}_{2} \mathrm{O}_{2}, E$. coli has one alkyl hydroperoxide reductase (Ahp) and two catalases (KatE and KatG) [5]. While much has been uncovered regarding how bacteria, such as $E$. coli, respond to $\mathrm{NO}$ and $\mathrm{H}_{2} \mathrm{O}_{2}$ treatment separately, less is known about how microbes respond to concurrent treatment. In a previous study, we investigated 
the response of $E$. coli to concurrent treatment with $\mathrm{NO}$ and $\mathrm{H}_{2} \mathrm{O}_{2}$ at concentrations reflective of phagosomal compartments $(\mu \mathrm{M})[11]$. We observed that $E$. coli prioritizes $\mathrm{H}_{2} \mathrm{O}_{2}$ elimination over $\mathrm{NO}$ in a dosedependent manner. Specifically, NO detoxification was significantly impaired by $\mathrm{H}_{2} \mathrm{O}_{2}$ (with larger doses corresponding to greater impairment), whereas $\mathrm{H}_{2} \mathrm{O}_{2}$ detoxification was unperturbed by $\mathrm{NO}$ at the concentrations investigated. A deeper analysis revealed that increasing doses of $\mathrm{H}_{2} \mathrm{O}_{2}$ impaired both transcription and translation of the major NO detoxification protein, Hmp, under aerobic conditions. Such a phenomenon has noticeable parallels with carbon catabolite repression (CCR), which occurs in environments with multiple carbon sources when microbes consume specific nutrients prior to others [12]. CCR has been widely observed across many bacterial species, with the preferred consumption of glucose over lactose by $E$. coli providing the prototypical example [12].

In this study, we were interested in exploring whether the prioritization of $\mathrm{H}_{2} \mathrm{O}_{2}$ over $\mathrm{NO}$, which we previously observed in $\mathrm{E}$. coli, was conserved across different bacterial species. In particular, we investigated dual stress conditions in Pseudomonas aeruginosa, which differs significantly from $E$. coli despite both being Gram-negative bacteria. P. aeruginosa inhabits very different niches in the human body, such as the airways and skin, compared to E. coli, which thrives in the gastrointestinal system [13, 14]. Genetically, $P$. aeruginosa and $E$. coli can harbor significantly different-sized genomes (e.g., $\sim 6.3 \cdot 10^{6}$ base pairs for $P$. aeruginosa PAO1, 4.6.10 6 base pairs for E. coli MG1655), whereas, metabolically, $P$. aeruginosa prefers a gluconeogenic metabolism (e.g., preferential consumption of succinate over glucose) and E. coli prefers a glycolytic metabolism (e.g., preferential consumption of glucose over succinate) [15-17]. Moreover, P. aeruginosa contains a different array of $\mathrm{NO}$ and $\mathrm{H}_{2} \mathrm{O}_{2}$ detoxification enzymes. Similar to $E$. coli, $P$. aeruginosa contains an NO dioxygenase (Fhp) and an NO reductase (NorCB), which are responsible for eliminating $\mathrm{NO}$ under aerobic and anaerobic conditions, respectively. However, NorCB uses a heme center for catalysis, whereas NorV uses a non-heme di-iron active site, and $P$. aeruginosa has a nitrite reductase (NirS) that generates $\mathrm{NO}$, while E. coli does not [18-21]. With regard to $\mathrm{H}_{2} \mathrm{O}_{2}$, P. aeruginosa possesses three alkyl hydro-peroxidases (AhpB, AhpC, Ohr) and three catalases (KatA, KatB, KatE), whereas $E$. coli contains one alkyl hydroperoxidase (AhpCF) and two catalases (KatG, KatE) [22]. For these reasons, we examined whether a similar prioritized detoxification of $\mathrm{H}_{2} \mathrm{O}_{2}$ and $\mathrm{NO}$ would be observed with P. aeruginosa.

\section{Main text}

Materials and methods

Bacterial strains

All experiments were performed using $P$. aeruginosa PAO1 (ATCC 15692).

\section{Chemicals and growth media}

All experiments were conducted in basal salts media (BSM) supplemented with $15 \mathrm{mM}$ succinate. The NO donor, (Z)-1-[N-(3-aminopropyl)-N-(3-ammoniopropyl) amino]diazen-1-ium-1,2-diolate (DPTA NONOate), was dissolved in $10 \mathrm{mM} \mathrm{NaOH}$ and stored on ice prior to use. $\mathrm{H}_{2} \mathrm{O}_{2}$ solution used was $35 \mathrm{wt}$. \% in water and was diluted to different stock concentrations $(10 \mathrm{mM}$ and $20 \mathrm{mM})$ in autoclaved Milli-Q water $\left(18.2 \mathrm{M} \Omega \mathrm{cm}\right.$ at $\left.25^{\circ} \mathrm{C}\right)$. LuriaBertani (LB) broth was made by dissolving LB powder in Milli-Q water and autoclaving the solution. Similarly, LB agar plates with pyruvate were made by dissolving LB powder and agar in Milli-Q water and autoclaving. After the solution had cooled, pyruvate was added at a concentration of $25 \mathrm{mM}$ and the solution was poured into sterile petri dishes. Pyruvate was used to scavenge any residual $\mathrm{H}_{2} \mathrm{O}_{2}$ from samples once plated.

\section{[NO] and $\left[\mathrm{H}_{2} \mathrm{O}_{2}\right]$ measurements}

Continuous measurement of NO concentrations was achieved using a $2 \mathrm{~mm}$ nitric oxide sensing probe (World Precision Instruments). The sensor was calibrated daily by adding increasing doses of SNAP (S-Nitroso-NAcetyl-D,L-Penicillamine) to $10 \mathrm{~mL}$ of $0.1 \mathrm{M} \mathrm{CuCl}_{2}$ solution per the manufacturer's instructions. A conversion factor of 0.457 molecules of NO per molecule of SNAP was used to convert calibration data to units of NO concentration [23]. $\mathrm{H}_{2} \mathrm{O}_{2}$ concentrations were determined using Amplex Red hydrogen peroxide/peroxidase kits (Life Technologies), per the manufacturer's instructions. Samples were diluted to less than $10 \mu \mathrm{M}$ and a standard curve with known concentrations $(0,1,2.5,5$ and $10 \mu \mathrm{M})$ was used to convert fluorescence values to $\mathrm{H}_{2} \mathrm{O}_{2}$ concentrations.

\section{[NO] and $\left[\mathrm{H}_{2} \mathrm{O}_{2}\right]$ consumption assays}

P. aeruginosa was taken from a $-80^{\circ} \mathrm{C}$ frozen stock, inoculated into $1 \mathrm{~mL}$ of LB media, and grown for $16 \mathrm{~h}$ in an incubator at $37{ }^{\circ} \mathrm{C}$ and 250 revolutions per minute (rpm). After $16 \mathrm{~h}$, the overnight culture was inoculated into $20 \mathrm{~mL}$ of BSM minimal media in a $250 \mathrm{~mL}$ baffled flask at an optical density at $600 \mathrm{~nm}\left(\mathrm{OD}_{600}\right)$ of 0.01 . The flask was incubated at $37{ }^{\circ} \mathrm{C}$ and $250 \mathrm{rpm}$ until cells reached mid-exponential phase $\left(\mathrm{OD}_{600} \sim 0.2\right)$. When the culture reached the desired $\mathrm{OD}_{600}, 8 \mathrm{~mL}$ of culture was transferred to 8 microcentrifuge tubes and spun at 15,000 rpm for $3 \mathrm{~min}$. After centrifugation, $980 \mu \mathrm{L}$ of supernatant 
was removed from each tube and cells were concentrated into $1 \mathrm{~mL}$ of BSM media. Before inoculation of cells into the bioreactor, $10 \mu \mathrm{L}$ of the appropriate stock solution of $\mathrm{H}_{2} \mathrm{O}_{2}$ was added to a bioreactor containing $10 \mathrm{~mL}$ of BSM media to reach a starting concentration of 10 or $20 \mu \mathrm{M}$. In assays performed in the absence of $\mathrm{H}_{2} \mathrm{O}_{2}, 10$ $\mu \mathrm{L}$ of autoclaved MilliQ water was added instead. Concentrated cell culture was added to bioreactors to achieve an initial $\mathrm{OD}_{600}$ of 0.025 . Immediately after inoculation, $6.95 \mu \mathrm{L}$ of $72 \mathrm{mM}$ DPTA NONOate was added to obtain an initial concentration of $50 \mu \mathrm{M}$ within the bioreactor, and the NO concentration was monitored continuously. In assays performed in the absence of $\mathrm{NO}$, the appropriate volume of $10 \mathrm{mM} \mathrm{NaOH}$ was added instead. At each time point, $150 \mu \mathrm{L}$ of solution was removed and sterile filtered using a $0.22 \mu \mathrm{M}$ syringe filter (Millex) to provide samples for $\mathrm{H}_{2} \mathrm{O}_{2}$ measurements. Samples for initial time points $(t=0)$ were removed prior to inoculation with cells. For assays lacking $\mathrm{H}_{2} \mathrm{O}_{2}, 150 \mu \mathrm{L}$ was removed at each time point to maintain equivalent reactor volumes throughout the assay.

\section{Cell culturability measurements}

To measure cell culturability, $200 \mu \mathrm{L}$ of solution was removed at time points, transferred to microcentrifuge tubes, and spun at $15,000 \mathrm{rpm}$ for $3 \mathrm{~min}$. Afterwards, $180 \mu \mathrm{L}$ of supernatant was removed, and the cell pellet was re-suspended in $980 \mu \mathrm{L}$ of phosphate buffered saline (PBS). The samples were then serially diluted in PBS and plated on LB agar supplemented with $25 \mathrm{mM}$ pyruvate. Plates were incubated at $37^{\circ} \mathrm{C}$ for $16 \mathrm{~h}$ at which time colonies were counted.

\section{Mathematical modelling}

The model used was constructed in previous studies [6, 21, 24-29]. For this study, however, the model was simplified and reduced to a system of only three ordinary differential equations to capture $\mathrm{NO}$ dynamics observed in a cell-free bioreactor upon delivery of $50 \mu \mathrm{M}$ of the NO donor DPTA NONOate, where $k_{\text {NONOate, }} k_{\text {autox }}, k_{L a, N O}$ and $k_{L a, O 2}$ are rate constants for NONOate degradation, $\mathrm{NO}$ autoxidation, $\mathrm{NO}$ mass transfer, and $\mathrm{O}_{2}$ mass transfer, respectively. $\left[\mathrm{O}_{2}\right]_{\text {sat }}$ refers to the dissolved oxygen concentration in equilibrium with air, whereas $\left[\mathrm{O}_{2}\right],[\mathrm{NO}]$, and [DPTA] refer to the $\mathrm{O}_{2}, \mathrm{NO}$, and DPTA NONOate concentrations within the bioreactor.

$$
\begin{aligned}
& \frac{d\left[\mathrm{O}_{2}\right]}{d t}=k_{L a, \mathrm{O} 2} \cdot\left(\left[\mathrm{O}_{2}\right]_{\text {sat }}-\left[\mathrm{O}_{2}\right]\right)-k_{\text {autox }} \cdot\left[\mathrm{NO}^{2}\left[\mathrm{O}_{2}\right]\right. \\
& \frac{d[\text { NONOate }]}{d t}=-k_{\text {NONOate }} \cdot[\text { NONOate }]
\end{aligned}
$$

\section{Parameter fitting}

Parameters were fit based on experimental data performed in a cell-free bioreactor dosed with $50 \mu \mathrm{M}$ DPTA NONOate. Specifically, the initial concentration of NO was set to zero, DPTA NONOate was set to $50 \mu \mathrm{M}$, and both $\left[\mathrm{O}_{2}\right]_{\text {sat }}$ and the initial $\left[\mathrm{O}_{2}\right]$ were set to $210 \mu \mathrm{M}$. The value for $k_{\mathrm{La}, \mathrm{O} 2}$ was obtained from a previous study using an identical apparatus [21]. The remaining parameters $\left(k_{\text {NONOate, }} k_{\text {autox }}, k_{L a, N O}\right)$ were optimized using a non-linear least squares regression algorithm (lsqcurvefit) that minimized the sum of the squared residual errors (SSR) between measured data and simulation data. One hundred initializations were performed using randomized initial values within previously established bounds [27]. Evidence ratios (ER) were calculated, and all parameters sets with an ER less than 10 were accepted as viable. Sixty-eight parameters sets were retained and a comparison between measured data and simulations performed with the optimal set $(E R=1)$ is plotted in Additional file 1: Figure S1A.

\section{Black-box modelling}

Due to the large size of the ensemble and the tight clustering of viable parameter sets (Additional file 1: Figure S1B), only the optimal parameter set was used to estimate cellular consumption using a black-box model. At each time interval in the experimental data, simulations were performed to estimate the rate of $\mathrm{NO}$ generated by DPTA NONOate, the rate of NO loss by autoxidation, and mass transport of NO to the gas phase. These values were used to calculate the change in $\mathrm{NO}$ in that time interval that could be attributed to abiotic means, which was subtracted from $\mathrm{d}[\mathrm{NO}] / \mathrm{dt}$ from the experimental data to calculate the rate of $\mathrm{NO}$ consumption by cells. The procedure was carried out at all time points up to NO clearance, defined as [NO] less than or equal to $0.2 \mu \mathrm{M}$, and the cumulative consumption of $\mathrm{NO}$ over time was calculated. NO consumption rates were

$$
\frac{d[N O]}{d t}=2 \cdot k_{\text {NONOate }} \cdot[N O N O a t e]-2 \cdot k_{\text {autox }}[N O]^{2}\left[\mathrm{O}_{2}\right]-k_{L a, N O} \cdot[N O]
$$


estimated for each condition by fitting linear portions of curves with lines of best fit and computing the slopes (Additional file 2: Figure S2). The number of points to include when estimating the line of best fit was chosen based on the maximum number of points in which the SSR did not dramatically increase between consumption curves and the best-fit line.

\section{Results}

In this study, we explored the relationship between $\mathrm{H}_{2} \mathrm{O}_{2}$ and NO detoxification in $P$. aeruginosa. Experimental conditions were chosen to mirror our previous study on E. coli [11]. Specifically, P. aeruginosa cells were grown to exponential phase and introduced into a bioreactor at an $\mathrm{OD}_{600}$ of 0.025 . Immediately after addition of cells, an NO donor (DPTA NONOate) was added at a concentration of $50 \mu \mathrm{M}$ to the reactor, as well as different concentrations of $\mathrm{H}_{2} \mathrm{O}_{2}(0,10$, or $20 \mu \mathrm{M})$. Increasing concentrations of $\mathrm{H}_{2} \mathrm{O}_{2}$ delayed $\mathrm{NO}$ detoxification by cells in a dose-dependent fashion (Fig. 1a). Similar to what was observed in E. coli, NO was detoxified in a biphasic manner (Fig. 1b). Initial NO consumption rates were similar across all treatment conditions $(\sim 100 \mathrm{nmol}$ per hour). The second phase of consumption rates deviated somewhat across culture conditions, but were all over threefold higher than initial rates. The drastic increases in consumption rates were only observed after detoxification of $\mathrm{H}_{2} \mathrm{O}_{2}$. Moreover, $\mathrm{H}_{2} \mathrm{O}_{2}$ clearance was unaffected by the presence of $\mathrm{NO}$ (Fig. 1c). Further, the culturability of samples exposed to $\mathrm{NO}$ and combination treatments of $\mathrm{NO}$ and $\mathrm{H}_{2} \mathrm{O}_{2}$ were comparable (Fig. 1d). Overall, the data demonstrated that $P$. aeruginosa also prioritized detoxification of $\mathrm{H}_{2} \mathrm{O}_{2}$ over the detoxification of $\mathrm{NO}$.

\section{Discussion}

Numerous bacteria have defense systems for immune antimicrobials that help them propagate infections [3034]. Among those antimicrobials are $\mathrm{NO}$ and $\mathrm{H}_{2} \mathrm{O}_{2}$, which are capable of inducing widespread cytotoxic effects on phagocytized bacteria [7, 8]. In a previous study, we investigated how E. coli responds to simultaneous $\mathrm{NO}$ and $\mathrm{H}_{2} \mathrm{O}_{2}$ exposure, and discovered that it prioritizes $\mathrm{H}_{2} \mathrm{O}_{2}$ elimination over that of $\mathrm{NO}$ [11]. Further, we found that the phenomenon was regulated at both the transcriptional and translational levels, which was reminiscent of CCR $[11,12]$. In this study, we investigated whether prioritized detoxification translated to $P$. aeruginosa. Interestingly, we observed
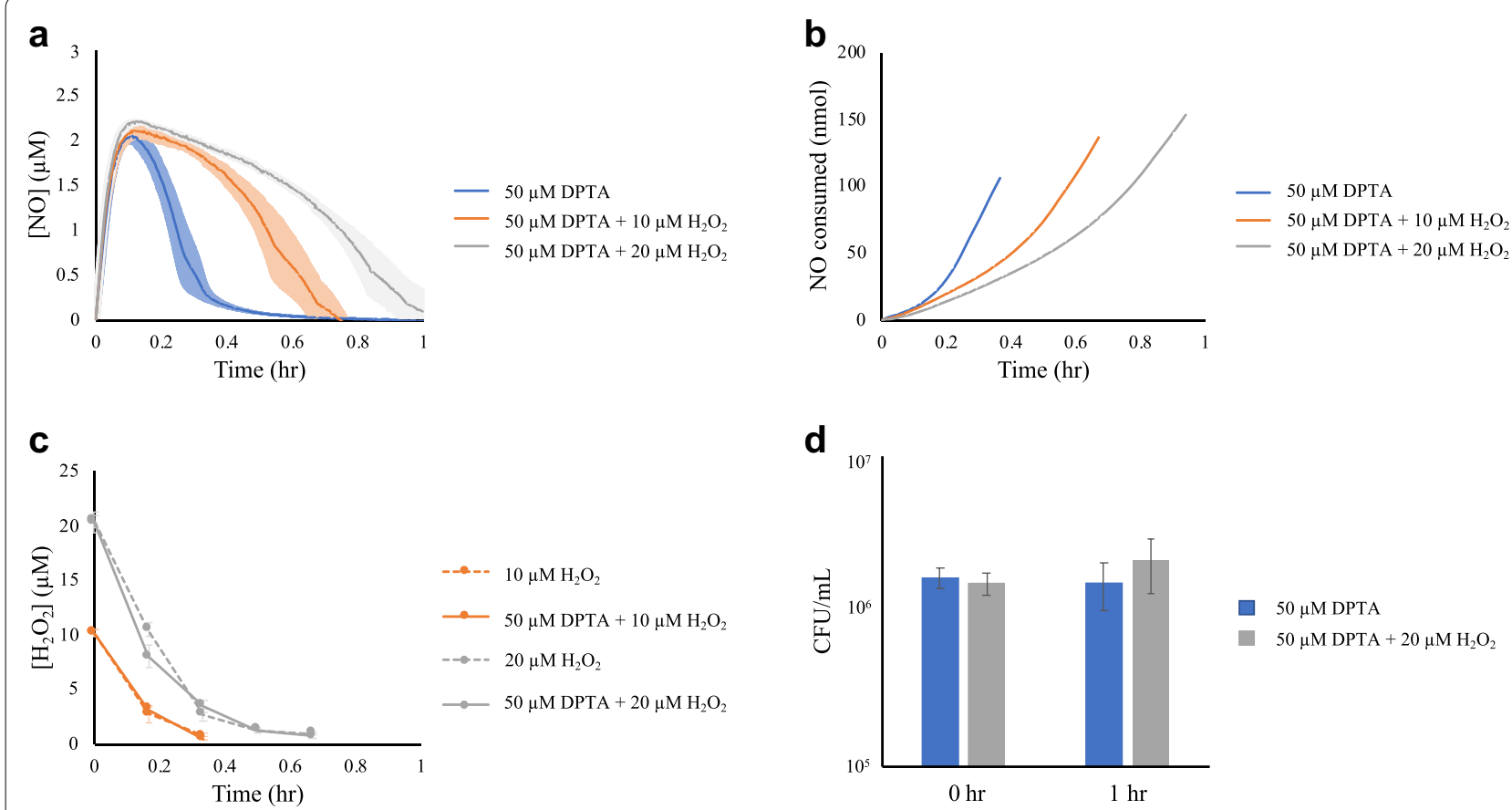

Fig. $1 \mathrm{H}_{2} \mathrm{O}_{2}$ clearance is prioritized over that of NO. P. aeruginosa cultures were grown to exponential phase and inoculated, at an $\mathrm{OD}_{600}$ of 0.025 , into a bioreactor containing either 0,10 , or $20 \mathrm{\mu M} \mathrm{H}_{2} \mathrm{O}_{2}$. Immediately after addition of cells, cultures were treated with either $50 \mu M$ DPTA NONOate or the same volume of the DPTA NONOate solvent. a NO concentrations in the bioreactor were continuously measured. b Cumulative cellular $\mathrm{NO}$ consumption was assessed using a kinetic model with a black-box cellular compartment. $\mathbf{c} \mathrm{H}_{2} \mathrm{O}_{2}$ concentrations were measured at 10-min intervals. $\mathbf{d}$ Culturability of $P$. aeruginos $a$ in the presence of $50 \mu \mathrm{M}$ DPTA and $50 \mu \mathrm{M}$ DPTA $+20 \mu \mathrm{M} \mathrm{H} \mathrm{H}_{2} \mathrm{O}_{2}$ were assessed at the beginning and $1 \mathrm{~h}$ after treatment. All data represents the mean of three replicates, with error bars representing the standard error of the mean 
that, similar to E. coli, $P$. aeruginosa $\mathrm{NO}$ detoxification was significantly delayed by cotreatment with $\mathrm{H}_{2} \mathrm{O}_{2}$, whereas $\mathrm{H}_{2} \mathrm{O}_{2}$ detoxification was unimpeded by $\mathrm{NO}$. Those results demonstrated that prioritized detoxification of these antimicrobials is not unique to $E$. coli and extends to other bacteria. Such a phenomenon may represent a highly conserved defensive strategy that bacteria use in multi-stress conditions, much like they use CCR in multi-nutrient conditions [12]. Looking forward, understanding the mechanistic bases of prioritized detoxification could lead to strategies to treat bacteria that use $\mathrm{NO}$ and $\mathrm{H}_{2} \mathrm{O}_{2}$ detoxification systems to enhance their virulence [9]. Such an anti-infective approach is currently being explored [35], along with other alternative treatments [36-40], with the ultimate goal of complementing currently available antibiotics.

\section{Limitations}

Further investigation into potential mechanisms for the prioritized detoxification in $P$. aeruginosa has not been performed. An assessment of Fhp transcription, translation, and catalytic activity under both $\mathrm{NO}$ and $\mathrm{NO}$ with $\mathrm{H}_{2} \mathrm{O}_{2}$ stress conditions will need to be evaluated.

\section{Abbreviations}

NO: Nitric oxide; $\mathrm{H}_{2} \mathrm{O}_{2}$ : Hydrogen peroxide; RNS: Reactive nitrogen species; CCR: Carbon catabolite repression; BSM: Basal salts media; DPTA NONOate: (Z)1-[N-(3-aminopropyl)-N-(3-ammoniopropyl)amino]diazen-1-ium-1,2-diolate; LB: Luria-Bertani; SNAP: S-Nitroso-N-Acetyl-D,L-Penicillamine; RPM: Revolutions per minute; $\mathrm{OD}_{600}$ : Optical density at $600 \mathrm{~nm}$; PBS: Phosphate buffered saline; SSR: Sum of square residuals; ER: Evidence ratios.

\section{Supplementary Information}

The online version contains supplementary material available at https://doi. org/10.1186/s13104-021-05534-7.

Additional file 1: Figure S1. Training of extracellular parameters. (A) Fifty $M$ M DPTA NONOate was added to a cell-free bioreactor and [NO] was continuously measured (blue line). The measured data is the mean of three replicates, with error bars representing the standard error of the mean. The data was used to train parameters in a kinetic model of NO reactivity and transport in the absence of cells. All parameter sets with $\mathrm{ER}<10$ were retained and considered viable sets. Due to the size of the ensemble and the tight clustering of parameter sets, simulations are plotted for only the optimal parameter set (minimum SSR, ER $=1$ ) (orange line). (B) A table containing the optimal, minimum, and maximum parameter values within the ensemble.

Additional file 2: Figure S2. Biphasic NO consumption rates under different treatment conditions. (A) $50 \mu \mathrm{M}$ DPTA. (B) $50 \mu \mathrm{M} \mathrm{DPTA}+10 \mu \mathrm{M}$ DPTA. (C) $50 \mu \mathrm{M}$ DPTA $+20 \mu \mathrm{M}$ DPTA. For each condition the rate of $\mathrm{NO}$ consumption for each regime was approximated by calculating the slope of the line of best fit. The equations of each line of best fit, and $R^{2}$ value are provided.

\section{Acknowledgements}

We would like to thank Weng Kang Chou for his assistance. Parameter optimizations were performed using the Terascale Infrastructure for Groundbreaking
Research in Science and Engineering (TIGRESS) high performance computing center at Princeton University, which is jointly supported by the Princeton Institute for Computational Science and Engineering (PICSciE) and the Princeton University Office of Information Technology's Research Computing department.

\section{Authors' contributions}

Experiments and analyses were designed by DMS and MPB. DMS performed the experiments and analyzed the data. The manuscript was written by DMS and MPB. All authors read and approved the final manuscript.

\section{Funding}

This work was supported by the Natural Sciences and Engineering Research Council of Canada (NSERC) and Princeton University (Helen Shipley Hunt Fund). The funders had no role in the preparation of the manuscript or decision to publish, and this content is solely the responsibility of the authors and does not necessarily represent the views of the funding agencies.

\section{Availability of data and materials}

The datasets generated for this study are available on request to the corresponding author.

\section{Declarations}

Ethics approval and consent to participate

Not applicable.

\section{Consent for publication}

Not applicable.

\section{Competing interests}

The authors declare that they have no competing interests.

\section{Author details}

1 Program in Quantitative and Computational Biology, Princeton University, Princeton, NJ, USA. ${ }^{2}$ Department of Chemical and Biological Engineering, Princeton University, Princeton, NJ, USA.

Received: 21 February 2021 Accepted: 16 March 2021

Published online: 26 March 2021

\section{References}

1. Flannagan RS, Cosío G, Grinstein S. Antimicrobial mechanisms of phagocytes and bacterial evasion strategies. Nat Rev Microbiol. 2009;7:355-66.

2. Flannagan RS, Jaumouillé V, Grinstein S. The cell biology of phagocytosis. Annu Rev Pathol Mech Dis. 2012;7:61-98.

3. Haas A. The phagosome: compartment with a license to kill. Traffic. 2007;8:311-30.

4. Fang FC. Antimicrobial reactive oxygen and nitrogen species: concepts and controversies. Nat Rev Microbiol. 2004;2:820-32.

5. Adolfsen KJ, Brynildsen MP. A kinetic platform to determine the fate of hydrogen peroxide in Escherichia coli. Rao CV, editor. PLoS Comput Biol. 2015;11:e1004562.

6. Robinson $J L$, Brynildsen MP. A Kinetic platform to determine the fate of nitric oxide in Escherichia coli. Rao CV, editor. PLoS Computational Biology. 2013;9:e1003049.

7. Wink DA, Mitchell JB. Chemical biology of nitric oxide: insights into regulatory, cytotoxic, and cytoprotective mechanisms of nitric oxide. Free Radical Biol Med. 1998;25:434-56.

8. Imlay JA. Pathways of oxidative damage. Annu Rev Microbiol. 2003;57:395-418.

9. Robinson JL, Adolfsen KJ, Brynildsen MP. Deciphering nitric oxide stress in bacteria with quantitative modeling. Curr Opin Microbiol. 2014;19:16-24.

10. Hrycay EG, Bandiera SM. Involvement of cytochrome P450 in reactive oxygen species formation and cancer. Adv Pharmacol. Elsevier; 2015 (cited 2021 Feb 17). p. 35-84. https://linkinghub.elsevier.com/retrieve/pii/ S1054358915000290 
11. Adolfsen KJ, Chou WK, Brynildsen MP. Transcriptional Regulation Contributes to Prioritized Detoxification of Hydrogen Peroxide over Nitric Oxide. MetcalfWW, editor. J Bacteriol. 2019;201:e00081-19, /jb/201/14/ JB.00081-19.atom.

12. Görke B, Stülke J. Carbon catabolite repression in bacteria: many ways to make the most out of nutrients. Nat Rev Microbiol. 2008;6:613-24.

13. Paulsson M, Su Y-C, Ringwood T, Uddén F, Riesbeck K. Pseudomonas aeruginosa uses multiple receptors for adherence to laminin during infection of the respiratory tract and skin wounds. Sci Rep. 2019:9:18168.

14. Blount ZD. The unexhausted potential of E. coli. ELife. 2015;4:e05826.

15. Blattner FR. The complete genome sequence of Escherichia coli K-12. Science. 1997;277:1453-62.

16. Stover CK, Pham XQ, Erwin AL, Mizoguchi SD, Warrener P, Hickey MJ, et al. PAO1, an opportunistic pathogen. 2000:406:7.

17. Rojo F. Carbon catabolite repression in Pseudomonas: optimizing metabolic versatility and interactions with the environment. FEMS Microbiol Rev. 2010;34:658-84.

18. Gomes CM, Giuffrè A, Forte E, Vicente JB, Saraiva LM, Brunori M, et al. A novel type of nitric-oxide reductase. J Biol Chem. 2002;277:25273-6.

19. Gardner AM, Helmick RA, Gardner PR. Flavorubredoxin, an inducible catalyst for nitric oxide reduction and detoxification in Escherichia coli. J Biol Chem. 2002;277:8172-7.

20. Hino T, Nagano S, Sugimoto H, Tosha T, Shiro Y. Molecular structure and function of bacterial nitric oxide reductase. Biochimica et Biophysica Acta (BBA) Bioenergetics. 2012;1817:680-7.

21. Robinson JL, Jaslove JM, Murawski AM, Fazen $\mathrm{CH}$, Brynildsen MP. An integrated network analysis reveals that nitric oxide reductase prevents metabolic cycling of nitric oxide by Pseudomonas aeruginosa. Metab Eng. 2017:41:67-81.

22. Heo Y-J, Chung I-Y, Cho W-J, Lee B-Y, Kim J-H, Choi K-H, et al. The major catalase gene (katA) of Pseudomonas aeruginosa PA14 Is under both positive and negative control of the global transactivator OxyR in response to hydrogen peroxide. JB. 2010;192:381-90.

23. Chou WK, Brynildsen MP. Loss of DksA leads to multi-faceted impairment of nitric oxide detoxification by Escherichia coli. Free Radical Biol Med. 2019:130:288-96

24. Robinson JL, Miller RV, Brynildsen MP. Model-driven identification of dosing regimens that maximize the antimicrobial activity of nitric oxide. Metabolic Eng Commun. 2014;1:12-8.

25. Robinson JL, Brynildsen MP. An ensemble-guided approach identifies ClpP as a major regulator of transcript levels in nitric oxide-stressed Escherichia coli. Metab Eng. 2015:31:22-34.

26. Robinson JL, Brynildsen MP. Discovery and dissection of metabolic oscil lations in the microaerobic nitric oxide response network of Escherichia coli. Proc Natl Acad Sci. 2016;113:E1757-66.
27. Sivaloganathan DM, Brynildsen MP. Quantitative modeling extends the antibacterial activity of nitric oxide. Front Physiol. 2020;11:330.

28. Sivaloganathan DM, Wan X, Brynildsen MP. Quantifying Nitric oxide flux distributions. In: Nagrath D, editor. Metabolic flux analysis in eukaryotic cells: methods and protocols. New York, NY: Springer US; 2020. p. 161-88. https://doi.org/10.1007/978-1-0716-0159-4_8

29. Robinson J, Brynildsen M. Construction and experimental validation of a quantitative kinetic model of nitric oxide stress in enterohemorrhagic Escherichia coli 0157:H7. Bioengineering. 2016:3:9.

30. Ehrt S, Schnappinger D. Mycobacterial survival strategies in the phagosome: defence against host stresses. Cell Microbiol. 2009;11:1170-8.

31. Hébrard M, Viala JPM, Méresse S, Barras F, Aussel L. Redundant hydrogen peroxide scavengers contribute to salmonella virulence and oxidative stress resistance. JB. 2009:191:4605-14

32. Shimizu T, Tsutsuki H, Matsumoto A, Nakaya H, Noda M. The nitric oxide reductase of enterohaemorrhagic Escherichia coli plays an important role for the survival within macrophages: the NO reductase of EHEC plays an important role for the survival within macrophages. Mol Microbiol. 2012;85:492-512.

33. Soares MP, Hamza I. Macrophages and iron metabolism. Immunity. 2016:44:492-504

34. Joo H-S, Fu C-I, Otto M. Bacterial strategies of resistance to antimicrobial peptides. Phil Trans R Soc B. 2016;371:20150292.

35. Chou WK, Vaikunthan M, Schröder HV, Link AJ, Kim H, Brynildsen MP Synergy screening identifies a compound that selectively enhances the antibacterial activity of nitric oxide. Front Bioeng Biotechnol. 2020;8:1001.

36. Usai D, Donadu M, Bua A, Molicotti P, Zanetti S, Piras S, et al. Enhancement of antimicrobial activity of pump inhibitors associating drugs. J Infect Dev Ctries. 2019;13:162-4.

37. Gupta V, Datta P. Next-generation strategy for treating drug resistant bacteria: Antibiotic hybrids. Indian J Med Res Wolters Kluwer Medknow. 2019;149:97-106.

38. Donadu M, Usai D, Pinna A, Porcu T, Mazzarello V, Fiamma M, et al. In vitro activity of hybrid lavender essential oils against multidrug resistant strains of Pseudomonas aeruginosa. J Infect Dev Ctries. 2018;12:009-14.

39. Escaich S. Antivirulence as a new antibacterial approach for chemotherapy. Curr Opin Chem Biol. 2008;12:400-8.

40. Dickey SW, Cheung GYC, Otto M. Different drugs for bad bugs: antivirulence strategies in the age of antibiotic resistance. Nat Rev Drug Discov. 2017;16:457-71.

\section{Publisher's Note}

Springer Nature remains neutral with regard to jurisdictional claims in published maps and institutional affiliations.
Ready to submit your research? Choose BMC and benefit from:

- fast, convenient online submission

- thorough peer review by experienced researchers in your field

- rapid publication on acceptance

- support for research data, including large and complex data types

- gold Open Access which fosters wider collaboration and increased citations

- maximum visibility for your research: over 100M website views per year

At BMC, research is always in progress.

Learn more biomedcentral.com/submissions 